\title{
Versuch, die Verdoppelung der auf der Marsobertläche beobachteten Linien auf optischem Wege zu erklären.
}

Von Ferdinand Meisel in Halle a. S.

Will man das Entstehen und Verschwinden eines zweiten, einem bereits vorhandenen Streifen benachbarten und parallelen Streifens auf der Marsoberfläche, wie Prof. Schiaparelli in Mailand es so vielfach beobachtet hat, als Neubildung, beziehungsweise Zerstörung eines wirklichen Objects auf dieser Oberfläche auffassen, so ist es ohne Ziveifel sehr schwierig, eine Erklärung hierfür zu finden, da jede Analogie mit irdischen Erscheinungen fehlt. Es liegt daher nahe, sich die Frage vorzulegen, ob sich die Verdoppelung der Marslinien vielleicht auf rein optischem Wege erklären, also auf die Entstehung eines doppelten Bildes zurückführen lässt. Wenn ich nun diese Frage bejahen zu dürfen glaube und einen solchen Erklärungsversuch hiermit den Fachkreisen vorlege, bitte ich vorher, denselben eben nur als einen Versuch betrachten zu wollen. Ich bin von dem Glauben weit entfernt, eine eigentliche 'Theorie der in Rede stehenden Erscheinung, zu welcher ja auch alle Daten fehlen würden, geliefert zu haben.

Da, wie Prof. Vogel schon vor Jahren spectroskopisch nachgewiesen hat, Mars eine an Wasserdampf sehr reiche Atmosphäre besitzt, wird sich an der Oberfläche des Planeten auch Wasser in tropfbar flüssigem Zustande finden, und es erscheint daher wohl kaum allzu gewagt, die Bezeichnungen s Meeres, ,Kanäles u. s. w., die Schiaparelli zunächst in rein topographischem Sinne gebraucht, auch in physikalischem Sinne anzuwenden, also anzunehmen, dass die -Meere wirkliche Wasseransammlungen, die , Kanälec wirkliche, nit Wasser gefüllte Rinnen seien. An der Oberfäche dieser mächtigen Wasserläufe muss nun eine ganz ausserordentlich starke Verdunstung stattfinden, viel stärker, als sie unter sonst gleichen Umständen an der Erdoberfläche stattfinden könnte. Ist auch die Intensität der Sonnenstrahlung auf dem Mars durchschnittlich im Verhältniss 0.4308 : I geringer, als auf der Erde, so ist andererseits die Masse des Mars nur etwa dem neunten Theil der Erdmasse gleich. Die geringere Masse hat eine in demselben Verhältnisse geringere Schwerkraft an der Oberfläche und diese wieder eine entsprechend geringere Dichtigkeit der Atmosphäre und also auch einen entsprechend geringeren Luftdruck zur Folge. Je geringer aber der Luftdruck ist, um so niedriger liegt bekanntlich der Siedepunkt des Wassers und um so intensiver ist bei beliebiger Temperatur die Dampfbildung. Aus diesem Grunde muss selbst bei der niedrigen Temperatur, welche auf dem Mars wahrscheinlich herrscht, eine sehr intensive Dampfentwickelung über den Wasserflächen stattfinden.

Die von der Oberfiäche eines Kanals aufsteigende Masse durchsichtigen Wasserdampfes vertheilt sich nun langsam in die Atmosphäre und breitet sich, da stets neuer Dampf erzeugt wird, nach oben und nach den Seiten aus; sie wird daher mit grösserer oder geringerer Regelmässigkeit die Gestalt eines halben Cylinders annehmen, dessen Axe etwa mit der Mittellinie des Wasserlaufs zusammenfällt. Tritt nun, durch irgend welche Vorgänge in der Atmosphäre veranlasst, -eine Ueberhöhung dieses halben Cylinders ein, ereignet es sich also, genauer ausgedrückt, dass der Krümmungshalbmesser des Cylinderquerschnitts im Scheitel kleiner als die Höhe des Scheitels Uber der Wasseroberfläche ist, so ist die Bedingung für die Entstehung einer Verdoppelung gegeben. Wie nachstehende Figur zeigt, können nämlich die vom Objecte $O$ - dem Kanalquerschnitt - ausgehenden Strahlen auf den verschiedenen Wegen $O A B$ und $O C D$ durch Brechung in das Fernrohr des irdischen Beobachters gelangen.

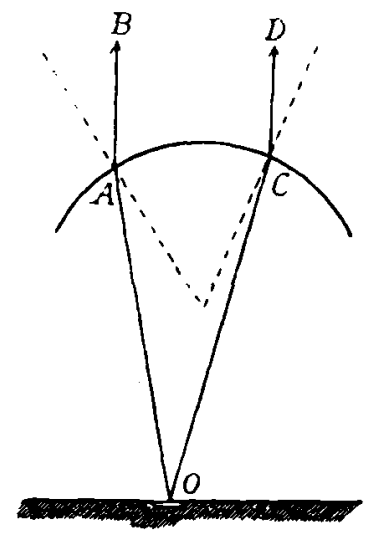

Der Abstand beider Bilder, sowie ihre Lage gegen das Object hängt, ausser von dem Brechungsvermögen des Dampfes, demjenigen der denselben umgebenden Luft, dem Krümmungsradius der Grenzfläche und ihrer Höhe über der Marsoberfläche, auch von der seitlichen Verschiebung des Scheitels der Grenzfläche gegen das Object $a b$, so dass sich daraus die verschiedenen Lagen der doppelten Linien gegen die nicht verdoppelte Fortsetzung desselben Kanals erklären. Ueberschreitet die Verschiebung ein gewisses Maass, so kann überhaupt nur noch e in Strahl die Richtang nach der Erde einschlagen, und die Verdoppelung verschwindet. Wird durch starke Bewegungen innerhalb der Atmosphäre die Dampfmasse noch weiter von der Mittellinie des Kanals entfernt, so kann es vorkommen, dass sämmtliche Strahlen, welche, von $O$ ausgehend, ungefähr die Richtung nach der Erde besitzen, unter so spitzem Winkel die Grenzfläche treffen, dass sie an dieser total reflectirt werden. In diesem Falle verschwindet also die Linie vollständig, was Professor Schiaparelli ebenfalls beobachtet hat.

Liegt die Scheitellinie der Grenzfläche genaı über der Axe des Kanals in der Richtung nach der Erde, so müssten eigentlich, da dann ein Strahl die brechende Fläche ungebrochen durchsetzt, drei Bilder entstehen, die indessen wahrscheinlich zu einem einzigen, breiteren, verschwommeneren Bilde sich vereinigen. Ueberhaupt dürfte diese exact geometrische Bedingung in Wirklichkeit kaum jemals erfüllt sein.

$\mathrm{Da}$ in Wirklichkeit eine scharfe Trennung zwischen der Dampfanhäufung und der umgebenden Atmosphäre 
zweifellos nicht besteht, der Wasserdampf vielmehr allnählich in die umgebende Atmosphäre übergehen und also auch die Dichtigkeit von der Wasseroberfäche aus allmählich abnehmen wird, muss auch die Richtungsänderung eine allmähliche sein. An die Stelle der gebrochenen Linien haben daher Curven zu treten, welche die Geraden $A B$ und $C D$ in $A$ und $C$ berüren. Am Wesen der Sache wird dadurch aber nichts geändert.

Dass es sich bei der Verdoppelung der Marslinien um eine rein optische Erscheinung handelt, wird durch manche der Beobachtungen Schiaparelli's noch besonders wahrscheinlich gemacht. So schreibt der genannte Forscher, dass die Farbe beider Streifen nach Art und Intensität inmer die gleiche ist, von einer zur anderen Verdoppelung jedoch erheblich wechseln kann, dass ferner, wenn ein doppelter Kanal durch einen anderen in zwei Abtheilungen zerlegt wird, und einer der Streifen breiter und heller auf der einen Seite des Schnittpunkts als auf der andern ist, es der andere Streifen auch ist; weiter, dass das Aussehen einer Verdoppelung mit der Zeit wechseln kann, dass die Verdoppelung in verhältnissmässig kurzer Zeit und in schnellem Wechsel stattfindet, und dass die beiden Kanäle sich oft aus einer Nebelmasse gleichzeitig loszulösen scheinen. Es scheint dem genannten Forscher fast, dass dieser nebehafte Zustand eine hauptsächliche Erscheinung bei der Bildung der Verdoppelungen sei.

Dass die Rotation des Mars um seine. Axe auf den Anblick der verdoppelten Linien keinen anderen Einfluss als den einer perspectivischen Verkürzung des Abstandes ausübt, wie mir Herr Professor Schiaparelli brieflich mitzutheilen die Güte hatte, ergiebt sich auch unschwer aus obiger Annahme. Den Querschnitt einer Dampfanhäufung, deren Krümmungshalbmesser im Scheitel kleiner als der Abstand des letzteren von der Marsoberfläche ist, wird man sich etwa parabolisch denken dürfen, so dass die Anhäufung die Gestalt eines parabolischen Cylinders mit zur Marsoberfläche normaler Symmetrie - Ebene haben würde. Dreht sich nun die so gestaltete Anhäufung von der Mitte der scheinbaren Scheibe aus nach der Seite, so treten dem irdischen Beschauer Punkte mit immer grösser werdendem Krümnungsradius gegenüber, so dass dementsprechend der Abstand der beiden Bilder continuirlich kleiner wird. Es findet also bei der Axendrehung des Planeten eine Aenderung des Abstandes beider Bilder statt, ganz ähnlich derjenigen, wie die perspectivische Verkürzung sie hervorbringen würde. Hierdurch wird natürlich die Täuschung, als handelte es sich um wirkliche, in der Marsoberfläche liegende Objecte, erhöht.

Man kann nun noch die Frage aufwerfen, weshalb denn nur die Kanäle, niemals aber die Ränder der Continente und die Inseln doppelt erscheinen. Auch dafür lässt sich, wie ich glaube, leicht ein Grund angeben.

Die gewaltige Dampfmasse, welche sich über einem Meere des Mars ohne Zweifel ansammelt, wird eine nahezu horizontale Oberfläche besitzen. Dieselbe wird sich nach dem Lande zu allerdings herabsenken, aber, da hier nicht ein intensives Nachströmen von einem eng begrenzten Striche aus erfolgt, wie das bei einem Kanale der Fall ist, wird auch die Senkung eine mehr allmähliche sein, so dass eine plötzlich eintretende, scharfe Krümmung der Oberfläche gerade oder nahezu uber der Uferlinie jedenfalls in hohem Grade unwahrscheinlich ist.

Wenn der in Obigem dargelegte Erklärungsversuch auch noch nicht auf alle Fragen Antwort giebt - so z. B. vermag ich für das Verschwinden der Unregelmässigkeiten bei der Verdoppelung noch keinen Grund einzusehen so glaube ich doch, dass das Wesentliche der merkwürdigen Erscheinung sich auf die angegebene Weise auf Grund anerkannter Naturgesetze erklären lässt. So lange über die Höhe der Marsatmosphäre und ihre Dichtigkeitsverhältnisse nichts bekannt ist, wird sich den Problem zwar rechnerisch nicht näher treten lassen, so dass die Frage, ob auf die angegebene Weise ein Abstand der Parallelen von mehreren Graden, wie er beobachtet wurde, erklärbar ist, zunächst eine offene bleibt, aber auch wohl kaum von vornherein verneint werden kann.

\section{Ueber die Wahrnehmung des Erdschattens ausserhalb der Mondscheibe. Von Dr. Hermann F. Klein.}

Die partiale Mondfinsterniss am Abend des 3. August 1887 wurde hier an einem sechsfüssigen Refractor bei 30 facher Vergrösserung beobachtet. Als zu Anfang der Verfinsterung der Mond aus den Dünsten trat und der Schatten schon merklich in die Scheibe vorgerückt war, sah ich zu meiner Verwunderung die Schattengrenze auch ausserhalb der Mondscheibe als bleigraue Wand, ähnlich einer Wolke, mit ganz scharfen Umrissen. Ein Mitbeobachter, Herr Tappen, bestätigte die Wahrnehmung. Die Grenze des Schattensegments war im Fernrohre so scharf, dass ich ihre Zenithdistanz wohl bis auf I' oder 2' genau hätte messen können. Diese Wahrnehmung wurde von $8^{\mathrm{h}} 15^{\mathrm{m}}$ bis $8^{\mathrm{h}} 30^{\mathrm{mm}}$ gemacht, später nicht mehr. Eine ähnliche Wahrnehmung ist meines Wissens vorher niemals gemacht worden. Später theilte mir Herr Dr. v. Konkoly mit, dass er bei derselben Finsterniss ganz die gleiche Erscheinung wahrgenommen habe. Gelegentlich der Finsterniss am 27. Januar dieses Jahres machte Herr E. Stuyvaert auf der Sternwarte zu Brüssel auch die Wahrnehmung, dass ein Segment des Erdschattens sich ausserhalb der Mondscheibe auf dem Himmelsgrunde zeigte. Zur Erklärung der 1887 von mir gesehenen Erscheinung hatte ich auf das von Mairan als Gegendämmerung bezeichnete Segment des Erdschattens in unserer Atmosphäre hingewiesen. Herr E. Stuyvaert hält dieser Erklärung entgegen, dass das Mairan'sche Segment unmöglich jene scharfe Begrenzung und Krümmung zeigen könne, welche in den bezeichneten Fällen sich dargestellt hat. Nach seiner Ansicht ist damals der Frdschatten auf irgend etwas Körperliches im Raume ausserhalb unserer Atmosphäre und sehr nahe beim Monde 